\title{
Interaction of Hydrogen with Palladium Clusters Deposited on Graphene
}

\author{
Julio A. Alonso a , Alejandra Granja ${ }^{a}$, Iván Cabria ${ }^{a}$ and María J. López ${ }^{\mathrm{a}}$ \\ ${ }^{a}$ Departamento de Física Teórica, Atómica y Optica, Universidad de Valladolid, 47011 Valladolid, Spain
}

\begin{abstract}
Hydrogen adsorption on nanoporous carbon materials is a promising technology for hydrogen storage. However, pure carbon materials do not meet the technological requirements due to the week binding of hydrogen to the pore walls. Experimental work has shown that doping with Pd atoms and clusters enhances the storage capacity of porous carbons. Therefore, we have investigated the role played by the Pd dopant on the enhancement mechanisms. By performing density functional calculations, we have found that hydrogen adsorbs on Pd clusters deposited on graphene following two channels, molecular adsorption and dissociative chemisorption. However, desorption of Pd-H complexes competes with desorption of hydrogen, and consequently desorption of Pd-H complexes would spoil the beneficial effect of the dopant. As a way to overcome this difficulty, Pd atoms and clusters can be anchored to defects of the graphene layer, like graphene vacancies. The competition between molecular adsorption and dissociative chemisorption of $\mathrm{H}_{2}$ on $\mathrm{Pd}_{6}$ anchored on a graphene vacancy has been studied in detail.
\end{abstract}

Keywords: Graphene; clusters; palladium; hydrogen adsorption.

PACS: 36.40.Jn; 82.33.Hk

\section{INTRODUCTION}

The successful storage of hydrogen in a light weight container is a problem that has to be solved to boost its use on electric cars powered by hydrogen fuel cells. However, the target of storing 5.5 weight $\%$ hydrogen at room temperature and pressures under $100 \mathrm{~atm}$ remains elusive, and efforts are being invested in different directions. A promising technology focusses on storing hydrogen in porous carbons, adsorbed on the pore walls. It appears that clean porous carbons do not meet the target, but doping the carbons with metallic atoms and nanoparticles enhances the amount of stored hydrogen, although the mechanisms for the enhancement have not been clarified. In a recent experimental work, Contescu et al. [1] have observed an enhancement in the hydrogen storage capacity of porous carbons doped with palladium. Therefore, it is of high interest to unravel the role played by the Pd dopant on the enhancement mechanisms. For this reason, we have studied the adsorption of hydrogen on small Pd clusters supported of carbon substrates, using density functional theory.

\section{PALLADIUM CLUSTERS ON GRAPHENE}

Massive computer simulations have shown that the walls of the pores in porous carbons have an atomic structure similar to that of graphene, although often curved and with defects [2]. For this reason, it is convenient to study the interaction of Pd clusters with graphene. Pd forms three-dimensional clusters on the graphene surface [3]. The strength of the bonding between the clusters and graphene is weak. However, the $\mathrm{Pd}_{\mathrm{n}}$ clusters become firmly anchored to vacancy defects existing in the pore walls, and this would solve some problems during the step of releasing the stored hydrogen to be used in the fuel cell [4].

There are two adsorption modes on palladium clusters supported on graphene. The first one is molecular adsorption, in which the bond length of the hydrogen molecule is slightly elongated, but not broken. The favored adsorption sites for the hydrogen molecules are on top of Pd atoms of the cluster. The second mode is dissociative chemisorption, in which the molecule dissociates and the two $\mathrm{H}$ atoms become chemisorbed with substantial bonding energies.

Specifically, we have investigated the competition between molecular adsorption and dissociative chemisorption for the case of hydrogen adsorption on the $\mathrm{Pd}_{6}$ cluster anchored to a graphene vacancy. The ground state structure of the anchored cluster, shown in Figure 1, is an octahedron resting on one of its triangular faces. One of the Pd atoms of that face sits above the center of the vacancy. A competitive structure is an incomplete bipyramid, in which one of the atoms of the pentagonal base is missing. This structure, also shown in Figure 1, can be viewed as an 
incomplete icosahedron. The total energy of this configuration is only $0.11 \mathrm{eV}$ higher than the energy of the anchored octahedral configuration.
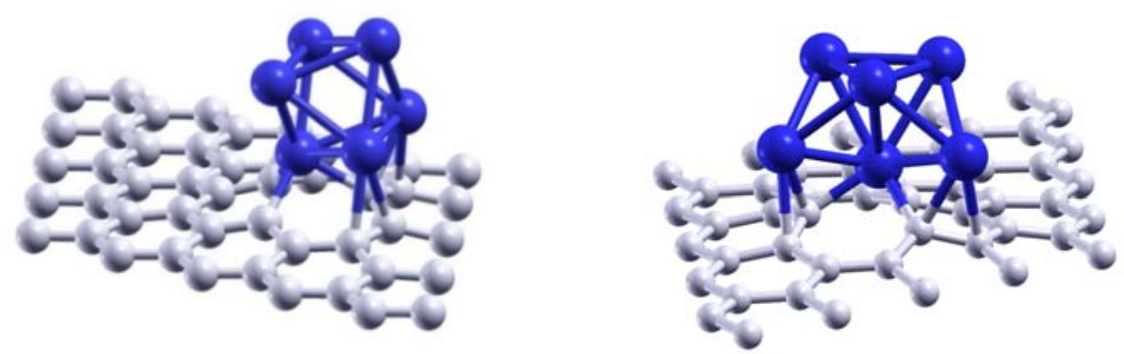

FIGURE 1. Octahedral and incomplete icosahedral structures of $\mathrm{Pd}_{6}$ adsorbed on a graphene vacancy. The icosahedral structure is less stable than the octahedral structure by $0.11 \mathrm{eV}$.

In the case of molecular adsorption on the anchored octahedral $\mathrm{Pd}_{6}$, the hydrogen molecules sit on sites on top of the $\mathrm{Pd}$ atoms of the cluster, excluding the Pd atom facing the center of the vacancy. The maximum number of $\mathrm{H}_{2}$ molecules directly adsorbed on the cluster is six, and the adsorption energies vary between 0.73 and $0.34 \mathrm{eV}$ as the number of molecules increases. This completes a first adsorption shell. Additional molecules can be adsorbed, but at longer distances from the cluster and with very low adsorption energies (see Figure 2). For instance the binding energy of a seventh molecule is $0.024 \mathrm{eV}$.

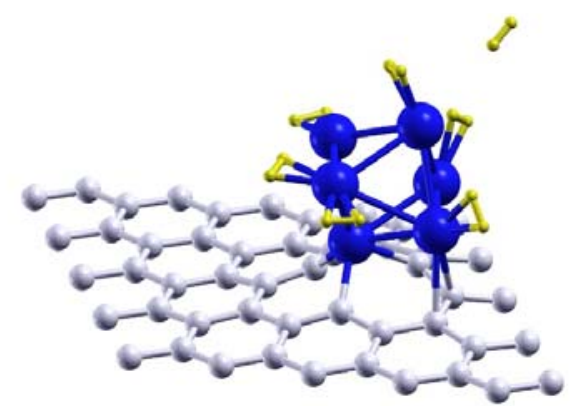

FIGURE 2. Seven hydrogen molecules adsorbed on $\mathrm{Pd}_{6}$ anchored on a vacancy in graphene. Six molecules form a first adsorption shell, and the seventh molecule rests at a longer distance and with a much lower binding energy than the others.

The lowest energy state of one adsorbed hydrogen molecule is, however, not the molecular state, but the dissociated state. Dissociation of the molecule in two $\mathrm{H}$ atoms has a dramatic effect on the $\mathrm{Pd}_{6}$ cluster, whose structure changes from the octahedral to the incomplete icosahedral configuration of Figure 1 . The chemisorption energy can be defined as

$$
E_{\text {chem }}=E\left(\mathrm{Pd}_{6} \text { on } \mathrm{G}_{\mathrm{vac}}\right)+E\left(\mathrm{H}_{2}\right)-E\left(2 \mathrm{H}+\mathrm{Pd}_{6} \text { on } \mathrm{G}_{\mathrm{vac}}\right) \text {, }
$$

where $E\left(\mathrm{Pd}_{6}\right.$ on $\left.\mathrm{G}_{\mathrm{vac}}\right)$ is the total energy of the system formed by the $\mathrm{Pd}_{6}$ cluster anchored on the graphene vacancy, $E\left(2 \mathrm{H}+\mathrm{Pd}_{6}\right.$ on $\left.\mathrm{G}_{\mathrm{vac}}\right)$ is the energy of the system formed by the dissociated molecule on the anchored $\mathrm{Pd}_{6}$ cluster, and $E\left(\mathrm{H}_{2}\right)$ is the energy of the isolated $\mathrm{H}_{2}$ molecule. The chemisorption energy amounts to $1.25 \mathrm{eV}$ if we take as reference the octahedral structure of the anchored $\mathrm{Pd}_{6}$, or $1.36 \mathrm{eV}$ if we take as reference the incomplete icosahedral structure. The difference accounts for the transformation energy from the octahedral to the icosahedral structure of anchored $\mathrm{Pd}_{6}$. The two $\mathrm{H}$ atoms take positions above two triangular faces of the incomplete bipyramid. Addition of more molecules results in a competition for adsorption sites and adsorption modes, molecular or dissociative. The second molecule is also dissociated, but in the case of the third added molecule the adsorption energies are very similar, $0.59 \mathrm{eV}$ for dissociative chemisorption and $0.58 \mathrm{eV}$ for molecular adsorption. Three molecules appear to 
saturate the power of the cluster to dissociate $\mathrm{H}_{2}$ and when the fourth, fifth, sixth and seventh molecules are added, these adopt the molecular adsorption mode on top of different Pd atoms. Figure 3 shows the case of five adsorbed molecules, three dissociated and two non-dissociated.

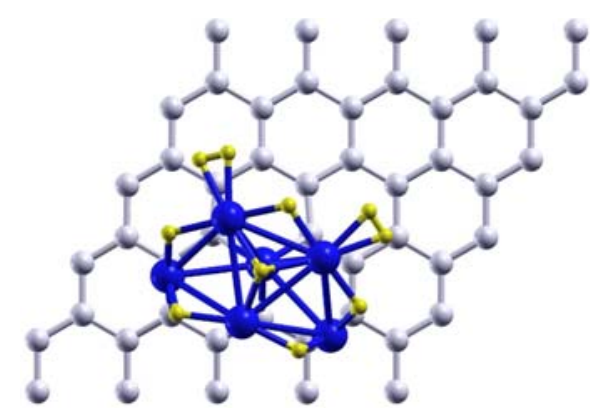

FIGURE 3. Adsorption of five hydrogen molecules on the incomplete pentagonal bipyramid structure of $\mathrm{Pd}_{6}$ anchored on a graphene vacancy. Three molecules are dissociated and two are in non-dissociated molecular form.

In summary, we have investigated the competition between the molecular adsorption and the dissociative chemisorption of hydrogen on the $\mathrm{Pd}_{6}$ cluster anchored on a graphene vacancy. The results are promising because the two mechanisms are expected to contribute to enhance the capacity of the material to adsorb hydrogen.

\section{ACKNOWLEDGMENTS}

Work supported by MICINN of Spain, (grant MAT2011-27881), and Junta de Castilla y León (Grant VA050U14).

\section{REFERENCES}

1. C. I. Contescu, K. van Benthem, S. Li, C. S. Bonifacio, S. J. Pennycook, P. Jena and N. C. Gallego, Carbon 49, 4050-4058 (2011).

2. M. J. López, I. Cabria and J. A. Alonso, J. Chem. Phys. 135, 104706:1-9 (2011).

3. I. Cabria, M. J. López and J. A. Alonso, Phys. Rev. B 81, 035403:1-5 (2010).

4. M. J. López, I. Cabria and J. A. Alonso, J. Phys. Chem. C 118, 5081-5090 (2014). 